\section{IN BRIEF}

\section{$\Rightarrow$ MICROBIOME}

\section{Leaving your polar bear host behind}

Many host-associated members of the microbiota are thought to have evolved from environmental relatives; by contrast, Welter et al. show that Psychrobacter spp. initially associated with animals and that some strains lost this ability and now are specialized to colonize cold environmental habitats. The authors generated 85 genomes of Psychrobacter spp. from different sources, including mammals, birds and fish, but also seawater, permafrost and ice. The genomic data revealed that the ancestors of the Psychrobacter clade are Moraxella spp., which are host-associated, often pathogenic bacteria. Furthermore, phenotypic screening of isolates revealed that there are two different ecotypes, those that are flexible and can grow in temperatures of up to $37^{\circ} \mathrm{C}$ and those with restricted growth that are limited to temperatures below $25^{\circ} \mathrm{C}$. Basal lineages belonged to the flexible ecotype and showed signs of host-association, such as bile resistance. Finally, although polar bear faeces contained strains of both ecotypes, only the flexible strains could colonize gnotobiotic mice. Thus, Psychrobacter spp. likely left host-associated niches and specialized to inhabit cold environments.

ORIGINAL ARTICLE Welter, D. K. et al. Free-Living, Psychrotrophic bacteria of the genus Psychrobacter are descendants of pathobionts. mSystems 6, e00258-21 (2021)

\section{ENVIRONMENTAL MICROBIOLOGY}

\section{Bacteria living on tree bark consume methane}

Trees and their environment, in particular wetlands, can emit substantial amounts of the greenhouse gas methane. Jeffrey et al. find that tree bark contains methane-oxidizing bacteria that can potentially mitigate some of these emissions. When they incubated bark samples of the common tree Melaleuca quinquenervia in the laboratory, they observed a reduction of methane concentration over time. The rate of methane consumption correlated with the abundance of methylotrophic bacteria (as determined by a metabolic marker gene), in particular members of the genus Methylomonas, which were common in the tree bark samples. Furthermore, field experiments with airtight chambers that can be used to measure methane fluxes revealed that the treatment of bark with difluoromethane, a competitive inhibitor of methantrophy, increased methane emissions by around $36 \%$.

ORIGINAL ARTICLE Jeffrey, L. C. et al. Bark-dwelling methanotrophic bacteria decrease methane emissions from trees. Nat. Commun. 12, 2127 (2021)

\section{VIRAL INFECTION}

\section{Blast from the past for coronavirus immunity}

Whether and how long it takes coronaviruses to evade host immunity is an important question. To study coronavirus antibody escape, Eguia et al. studied human serum samples from the 1980s and 1990s and their ability to neutralize the common cold coronavirus $229 \mathrm{E}$. They assessed the ability of the samples to neutralize viruses pseudotyped with spike from five $229 \mathrm{E}$ strains from different time points, ranging from 1986 to 2016. Sera that potently neutralized viruses with concomitant spikes showed a substantial drop in neutralization titres for later viruses or even complete escape, likely due to divergence of the receptor-binding domain. On average, 10 years of 'future' virus evolution led to a fourfold drop in neutralization titres. By contrast, sera collected in 2020 neutralized the 'old' viruses, at least if the sample came from an adult. Children's sera showed some neutralization of previous strains but not as far back as adult sera. ORIGINAL ARTICLE Eguia, R. T. et al. A human coronavirus evolves antigenically to escape antibody immunity. PLOS Pathog. 17, e1009453 (2021)

\title{
Change of programme
}

Integrative and conjugative elements (ICEs) are found in a diverse range of Gram-positive and Gram-negative bacteria. ICEs are integrated into host chromosomes, but following their activation, they can excise, circularize and be transferred to recipient cells via conjugation. In addition to the required conjugation machinery, ICEs often encode accessory genes that confer a distinct phenotype to host cells, including virulence factors and antibiotic or metal resistance, and the transfer of such mobile elements and the encoded traits between bacteria promotes the rapid evolution and spread of such phenotypes. Although many ICE-encoded genes have been identified bioinformatically, the phenotypes that they might confer to their hosts often remain unknown. In this study, Jones et al. report that ICEBs 1, which is an ICE of Bacillus subtilis, provides a selective advantage to its host cells during two developmental processes: biofilm formation and sporulation.

ICEBs 1 transfer is activated following the sensing of distinct signals, for example, high cell density stimulates the activation of ICEBs 1 , and ICEBs 1 transfer is repressed by a quorum-sensing signal encoded on the element. During growth in a biofilm, ICEBs 1 is induced and transfers efficiently. However, it was unknown whether ICE $B s 1$ affects host cells during this growth mode.

To determine the possible impact on the fitness of its host, the authors performed competition experiments in biofilms using strains of B. subtilis with or without ICEBs1. They found that ICEBs 1 efficiently spreads at low donor to recipient ratios during growth in biofilms, confirming previous observations showing that ICEBs 1 induction is inhibited by the presence of other potential donors. In addition,

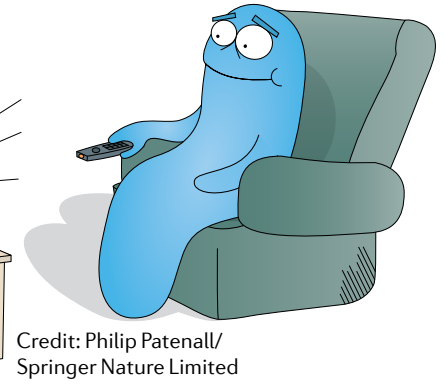

the authors report the enhanced fitness of ICEBs1-containing cells compared with cells that do not contain the ICEBs 1 , which was also dependent on the initial frequency of the element in the population. But what underlies this ICEBs1-mediated growth advantage?

Interestingly, the authors found that cells containing ICEBs1 'cheat' by decreasing the expression of biofilm matrix genes compared with cells without ICE $B s 1$, possibly enabling them to avoid the costly production of this public good. In addition, sporulation of ICEBs1-containing cells was delayed in a frequency-dependent manner both during growth in biofilms and in the absence of biofilms, thus enabling extended cell growth until committing to the developmental programme. The authors showed that the expression of the ICEBs I gene devI was necessary and sufficient to reduce biofilm matrix expression and delay the initiation of sporulation.

In summary, the study shows that delaying biofilm and spore development enables the ICEBs1containing host cells to grow more than cells without ICEBs1, which increases propagation of the element. Thus, ICEBs1-encoded genes modulate normal host functions rather than provide entirely new phenotypes to their hosts, which the authors speculate might be a conserved strategy among ICEBs1-like elements or other ICEs.

Andrea Du Toit

ORIGINAL ARTICLE Jones, J. M. et al. A mobile genetic element increases bacterial host fitness by manipulating development. eLife 10, e65924 (2021) 